\title{
Edge chromatic 6-critical graphs
}

\author{
J. Sakila Devi
}

\begin{abstract}
In this paper, we study the structure of edge chromatic 6-critical graphs of three different classes by obtaining the bounds on the number of major vertices and the size.
\end{abstract}

Keywords

Class one, class two, edge colouring, edge chromatic critical graphs.

AMS Subject Classification

05C15, $05 \mathrm{C} 75$.

Department of Mathematics, Lady Doak College, Madurai-625002, Tamil Nadu, India.

*Corresponding author: sakiladevi@Idc.edu.in

Article History: Received 21 December 2018; Accepted 11 February 2019

(C)2017 MJM.

\section{Contents}

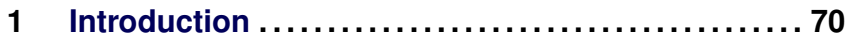

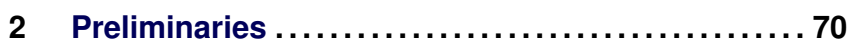

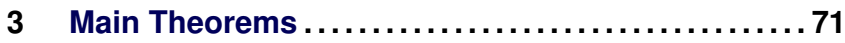

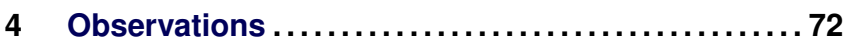

5 Conclusion $\ldots \ldots \ldots \ldots \ldots \ldots \ldots \ldots \ldots \ldots \ldots \ldots, 72$

References ................................. 72

\section{Introduction}

Throughout this paper, Let $G=(V, E)$ be a simple graph with $n$ vertices and $m$ edges. The degree of a vertex $v$ in $G$ is denoted by $d(v)$. A $k$-vertex in $G$ is a vertex of degree $k$ in $G$. Let $\Delta(G), \delta(G)$ (or $\Delta, \delta$ ) be the maximum degree and the minimum degree of $G$. A vertex $v$ with $d(v)=\Delta$ is called a major vertex. A vertex $v$ with $d(v)<\Delta$ is called a minor vertex. Let $N(x)$ be the set of all neighbours of $x$ in $G$ and $N(x, y)=N(x) \bigcup N(y)$. If $S$ and $T$ are disjoint subsets of $V(G)$, then $[S, T]$ denotes the set of all edges with one end in $S$ and the other in $T$. The subgraph of G induced by $S$ is denoted by $[S]$ and $|[S]|$ denotes $|E[S]|$.The number of vertices of degree $j$ is denoted by $n_{j}$. The degree sequence of $\mathrm{G}$ is denoted by $\pi(G)=1^{n_{1}} 2^{n_{2}} \ldots \Delta^{n_{\Delta}}$, where if $n_{j}=0$, then the factor $j^{n_{j}}$ is customarily omitted in $\pi(G)$. An edge colouring of a graph is a function assigning colours to the edges of the graph in such a way that any two adjacent edges receive different colours. A graph $G$ is edge $k$-colourable, if there is an edge colouring of the graph $G$ with colours from $\{1, \ldots, k\}$. The edge chromatic number or chromatic index of a graph $G$, denoted by $\chi^{\prime}(G)$, is the smallest integer $k$ such that $G$ is edge $k$-colourable. In 1964, Vizing[8] proved that if $G$ is a simple graph of maximum degree $\Delta$, then the edge chromatic number $\chi^{\prime}(G)$ of $G$ is either $\Delta$ or $\Delta+1$. If $\chi^{\prime}(G)=\Delta(G), G$ is said to be of class one; otherwise $G$ is said to be of class two. A graph $G$ is said to be edge chromatic critical if $G$ is connected, of class 2 and $\chi^{\prime}(G-e)<\chi^{\prime}(G)$ for every edge $e$ of $G$. If $G$ is critical and $\Delta(G)=\Delta$, then $G$ is said to be $\Delta$-critical.

\section{Preliminaries}

A well known fundamental result commonly known as Vizing's Adjacency Lemma(VAL) describes an important property of $\Delta$ - critical graphs.

R1: Vizing's Adjacency Lemma[9](VAL): In a $\Delta$-critical graph $\mathrm{G}$, if $v w$ is an edge and $d(v)=k$, then $w$ is adjacent with at least $\Delta-k+1$ other vertices of degree $\Delta$. In particular, $\mathrm{G}$ has at least $\Delta-\delta+2$ vertices of degree $\Delta$.

R2: Fiorini's Inequality[1]: Let $\Delta \geq 3$ be an integer. If $\mathrm{G}$ is a $\Delta$-critical graph, then $n_{\Delta} \geq 2 \sum_{j=2}^{n-1} \frac{n_{j}}{j-1}$.

R3:[6] Let $\Delta \geq 4$ be an integer. If $\mathrm{G}$ is a $\Delta$-critical graph, then $n_{\Delta} \geq 2 \sum_{j=2}^{\Delta-1} \frac{n_{j}}{j-1}+\frac{1}{2} n_{3}$.

R4: Vizing's Conjecture[10]: If $\mathrm{G}$ is a $\Delta$-critical simple graph with $n$ vertices, $m$ edges and $\Delta \geq 3$, then $m \geq \frac{1}{2}((\Delta-$ 1) $n+3)$.

This conjecture has been verified for the critical graphs with $\Delta \leq 6[2,3,4,7]$.

R5:( Zhang [5] ) Let $G$ be a $\Delta$ - critical graph, $x y \in E(G)$ and $d(x)+d(y)=\Delta+2$. Then the following hold: (i) every vertex of $N(x, y)-\{x, y\}$ is a major vertex (ii) every vertex of $N(N(x, y))-\{x, y\}$ is at least of degree $\Delta-1$ (iii) If 
$d(x), d(y)<\Delta$, then every vertex of $N(N(x, y))-\{x, y\}$ is a major vertex.

\section{Main Theorems}

In this section, we study the structure of edge chromatic 6-critical graphs of three different classes. Throughout this section, we use the following notations: $M$ denotes the set of all major vertices of $G$ and $N_{2}, N_{3}, N_{4}, N_{5}$ are the sets of all vertices of degree two, three, four and five respectively and $M_{2}, M_{3}, M_{4}, M_{5}, M_{34}, M_{35}, M_{45}, M_{345}$ denote the sets of all major vertices adjacent with minor vertices which are only 2 -vertices, only 3 -vertices, only 4 -vertices, only 5 -vertices, 3 and 4 -vertices, 3 and 5-vertices, 4 and 5-vertices, 3, 4, and 5 -vertices respectively. In the following theorems, we have obtained bounds on the number of major vertices and the size of three classes of edge chromatic 6-critical graphs, that are stronger than the existing bounds.

Theorem 3.1. If $G$ is a 6-critical graph in which each 3-vertex is adjacent with a 5-vertex and each 4-vertex is adjacent with another $4-v e r t e x$, then $n_{6} \geq 2 n_{2}+\frac{9}{2} n_{3}+\frac{3}{2} n_{4}+\frac{1}{2} n_{5}$, $m \geq \frac{5}{2} n-\frac{1}{2} n_{2}+\frac{5}{4} n_{3}+\frac{1}{4} n_{4}+\frac{1}{4} n_{5}$ and hence $m>\frac{5}{2} n+\frac{3}{2}$ if $5 n_{3}+n_{4}+n_{5}-2 n_{2}>6$.

Proof. Since each 4-vertex is adjacent with another 4-vertex and each 3-vertex is adjacent with a 5-vertex , by $R 5, M_{45}=\phi$ and $M_{34}=\phi$. By VAL, $M_{345}=\phi$. Let $\left|\left[M_{35}, N_{3}\right]\right|=k$. By VAL, each vertex in $M_{35}$ is adjacent with exactly one 3-vertex and exactly one 5-vertex. Thus, $\left|M_{35}\right|=k$. Since every 3vertex is adjacent with a 5-vertex, by VAL, $\left|\left[N_{3}, N_{5}\right]\right|=n_{3}$. Since each 4-vertex is adjacent with a 4-vertex, by VAL, $\left|\left[N_{4}, N_{5}\right]\right|=0$. Let $N_{5}^{\prime}$ be the set of all 5-vertices which are not adjacent with any 3-vertices. Let $N_{5}^{\prime \prime}=N_{5}-N_{5}^{\prime}$. Each vertex in $N_{5}^{\prime \prime}$ is adjacent with exactly one 3-vertex and hence $\left|N_{5}^{\prime \prime}\right|=n_{3}$ and $\left|N_{5}^{\prime}\right|=n_{5}-n_{3}$. By VAL, each vertex in $N_{5}^{\prime \prime}$ has four major neighbours. Thus $\left|\left[N_{5}^{\prime \prime}\right]\right|=0$ and $\left|\left[N_{5}^{\prime \prime}, N_{5}^{\prime}\right]\right|=0$. Also, by R5, $\left|\left[N_{5}^{\prime}, M_{35}\right]\right|=0$. Thus by VAL, $\left|\left[N_{5}^{\prime}, M_{5}\right]\right| \geq 2\left(n_{5}-n_{3}\right)$. By R5, each vertex in $M_{5}$ can be adjacent with at most one vertex in $N_{5}^{\prime \prime}$ and a vertex in $M_{5}$ can be adjacent with at most four vertices in $N_{5}^{\prime}$. Thus $\left|\left[N_{5}^{\prime \prime}, M_{5}\right]\right|=4 n_{3}-k$ and each vertex of these $4 n_{3}-k$ vertices have five major neighbours. Thus, $\left|M_{5}\right| \geq\left|\left[N_{5}^{\prime \prime}, M_{5}\right]\right|+\frac{\left|\left[N_{5}^{\prime}, M_{5}\right]\right|}{4},\left|M_{5}\right| \geq 4 n_{3}-k+\frac{1}{2}\left(n_{5}-n_{3}\right)$ and it implies that $\left|M_{5}\right| \geq \frac{7}{2} n_{3}+\frac{1}{2} n_{5}-k$. Since every $4-$ vertex is adjacent with a 4-vertex, by VAL, $\left|\left[N_{4}\right]\right|=\frac{n_{4}}{2}$. Also, by VAL, $\left[N_{4}, N_{3}\right]=\phi$. Thus $\left|\left[N_{4}, M_{4}\right]\right|=4 n_{4}-2\left|\left[N_{4}\right]\right|=4 n_{4}-n_{4}=$ $3 n_{4}$. By R5, each vertex in $M_{4}$ is adjacent with at most two 4-vertices and each vertex in $M_{4}$ has at least four major neighbours. Hence $\left|M_{4}\right| \geq \frac{1}{2}\left|\left[N_{4}, M_{4}\right]\right|=\frac{3}{2} n_{4}$. Now $\left|\left[N_{3}, M_{3}\right]\right|=$ $3 n_{3}-\left|\left[N_{3}, N_{5}\right]\right|-\left|\left[N_{3}, M_{35}\right]\right|=2 n_{3}-k$. Since each 3-vertex is adjacent with a 5-vertex, by R5, every vertex in $M_{3}$ has exactly five major neighbours. Thus each vertex in $M_{3}$ is adjacent with exactly one 3 -vertex. Thus $\left|M_{3}\right|=\left|\left[N_{3}, M_{3}\right]\right|=2 n_{3}-k$. Also by VAL, we observe that $\left|\left[N_{2}, M_{2}\right]\right|=2 n_{2}$ and $\left|M_{2}\right|=2 n_{2}$. Now $|M| \geq\left|M_{2}\right|+\left|M_{3}\right|+\left|M_{4}\right|+\left|M_{5}\right|+\left|M_{35}\right| \geq 2 n_{2}+2 n_{3}-$ $k+\frac{3}{2} n_{4}+\frac{7}{2} n_{3}+\frac{1}{2} n_{5}$. (i.e) $n_{6} \geq 2 n_{2}+\frac{11}{2} n_{3}+\frac{3}{2} n_{4}+\frac{1}{2} n_{5}-k$.
Since $k \leq n_{3}, n_{6} \geq 2 n_{2}+\frac{9}{2} n_{3}+\frac{3}{2} n_{4}+\frac{1}{2} n_{5}$. Now $2 m=2 n_{2}+$ $3 n_{3}+4 n_{4}+5 n_{5}+6 n_{6}=5 n-3 n_{2}-2 n_{3}-n_{4}+n_{6} \geq 5 n-$ $3 n_{2}-2 n_{3}-n_{4}+2 n_{2}+\frac{9}{2} n_{3}+\frac{3}{2} n_{4}+\frac{1}{2} n_{5}=5 n-n_{2}+\frac{5}{2} n_{3}+$ $\frac{1}{2} n_{4}+\frac{1}{2} n_{5}$. Thus $m \geq \frac{5}{2} n-\frac{1}{2} n_{2}+\frac{5}{4} n_{3}+\frac{1}{4} n_{4}+\frac{1}{4} n_{5}$. Thus if $5 n_{3}+n_{4}+n_{5}-2 n_{2}>6$, then $m>\frac{5}{2} n+\frac{3}{2}$. Note that, if $n_{2}=0$ and $n_{3}=n_{5}$, then $m \geq \frac{5}{2} n+\frac{3}{2} n_{5}+\frac{1}{4} n_{4}$.

Theorem 3.2. If $G$ is a 6-critical graph such that each 4vertex is adjacent with another 4-vertex and no 3-vertex is adjacent with a 5-vertex, then $n_{6} \geq 2 n_{2}+\frac{3}{2} n_{3}+\frac{3}{2} n_{4}+\frac{1}{2} n_{5}+\frac{k}{4}$, $m \geq \frac{5}{2} n-\frac{1}{2} n_{2}-\frac{1}{4} n_{3}+\frac{1}{4} n_{4}+\frac{1}{4} n_{5}+\frac{k}{8}$ and hence $m>\frac{5}{2} n+\frac{3}{2}$ if $2 n_{5}+2 n_{4}-2 n_{3}-4 n_{2}+k>12$ where $k=\left|\left[M_{35}, N_{3}\right]\right|$.

Proof. Since each 4-vertex is adjacent with another 4-vertex, by R5, $M_{45}=M_{34}=\phi$. Also, by VAL, $M_{345}=\phi$ and each vertex in $M_{35}$ is adjacent with exactly one 3-vertex and exactly one 5-vertex. Thus $\left|M_{35}\right|=k$. Since each 4 -vertex is adjacent with a 4-vertex, by VAL, $\left|\left[N_{4}, N_{5}\right]\right|=0$ and $\left|\left[N_{4}, N_{3}\right]\right|=0$. Thus $\left|\left[N_{4}, M\right]\right|=3 n_{4}$. Since $\left|\left[M_{35}, N_{3}\right]\right|=\left|\left[M_{35}, N_{5}\right]\right|=k$, $\left|\left[N_{3}, M_{3}\right]\right|=3 n_{3}-k$. By VAL , $\left|M_{2}\right|=2 n_{2}$. Since $M_{45}=$ $M_{34}=\phi,\left|\left[N_{4}, M_{4}\right]\right|=3 n_{4}$. Since each 5-vertex is adjacent with at least two major vertices and $\left|\left[N_{5}, M_{35}\right]\right|=k$, we get that $\left|\left[N_{5}, M_{5}\right]\right| \geq 2 n_{5}-k$. Since a 5 -vertex can be adjacent with at most three 5 -vertices, $\left|\left[N_{5}\right]\right| \leq \frac{3 n_{4}}{2}$. Since each vertex in $M_{3}$ can be adjacent with at most two 3-vertices, $\left|M_{3}\right| \geq$ $\frac{3 n_{3}-k}{2}$. Since each vertex in $M_{4}$ can be adjacent with at most two 4-vertices, $\left|M_{4}\right| \geq \frac{3 n_{4}}{2}$. Each vertex in $M_{5}$ can be adjacent with at most four 5 -vertices. So, $\left|M_{5}\right| \geq \frac{2 n_{5}-k}{4}$. Now $|M| \geq\left|M_{2}\right|+\left|M_{3}\right|+\left|M_{4}\right|+\left|M_{5}\right|+\left|M_{35}\right| \geq 2 n_{2}+\frac{3}{2} n_{3}-\frac{k}{2}+$ $\frac{3}{2} n_{4}+\frac{1}{2} n_{5}-\frac{k}{4}+k$. (i.e) $n_{6} \geq 2 n_{2}+\frac{3}{2} n_{3}+\frac{3}{2} n_{4}+\frac{1}{2} n_{5}+\frac{k}{4}$. Now $2 m=2 n_{2}+3 n_{3}+4 n_{4}+5 n_{5}+6 n_{6}=5 n-3 n_{2}-2 n_{3}-$ $n_{4}+n_{6} \geq 5 n-3 n_{2}-2 n_{3}-n_{4}+2 n_{2}+\frac{3}{2} n_{3}+\frac{3}{2} n_{4}+\frac{1}{2} n_{5}+\frac{k}{4}$. Thus $2 m \geq 5 n-n_{2}+\frac{1}{2} n_{3}+\frac{1}{2} n_{4}+\frac{1}{2} n_{5}+\frac{k}{4}$. Hence $m \geq \frac{5}{2} n-$ $\frac{1}{2} n_{2}-\frac{1}{4} n_{3}+\frac{1}{4} n_{4}+\frac{1}{4} n_{5}+\frac{k}{8}$. So, $m>\frac{5}{2} n+\frac{3}{2}$ if $2 n_{5}+2 n_{4}-$ $2 n_{3}-4 n_{2}+k>12$.

Theorem 3.3. If $G$ is a 6-critical graph such that every 3vertex is adjacent with a 5-vertex, no 4-vertex is adjacent with any minor vertices and no major vertex is adjacent with both 4-vertex and 5-vertex, then $n_{6} \geq 2 n_{2}+\frac{3}{2} n_{3}+\frac{4}{3} n_{4}+\frac{1}{2} n_{5}+\frac{k}{4}$, $m \geq \frac{5}{2} n-\frac{1}{2} n_{2}-\frac{1}{4} n_{3}+\frac{1}{6} n_{4}+\frac{1}{4} n_{5}+\frac{k}{8}$ and hence $m>\frac{5}{2} n+\frac{3}{2}$ if $4 n_{4}+6 n_{5}-12 n_{2}-6 n_{3}+3 k>36$ where $k=\left|\left[M_{35}, N_{3}\right]\right|$.

Proof. Since every 3-vertex is adjacent with a 5-vertex, by $\mathrm{R} 5, M_{34}=M_{345}=\phi$. Since $\left|\left[N_{3}, M_{35}\right]\right|=k$ and each vertex in $M_{35}$ is adjacent with exactly one 3-vertex and one 5-vertex, $\left|M_{35}\right|=k$ and $\left|\left[M_{35}, N_{5}\right]\right|=k$. Hence by VAL, $\left|\left[N_{3}, M_{3}\right]\right|=$ $2 n_{3}-k$. Each vertex in $M_{3}$ can be adjacent with at most two 3vertices. Thus $\left|M_{3}\right| \geq \frac{2 n_{3}-k}{2}=n_{3}-\frac{k}{2}$. Since no 4-vertex is adjacent with any minor vertex, $\left|\left[N_{4}\right]\right|=\left|\left[N_{4}, N_{5}\right]\right|=\left|\left[N_{4}, N_{3}\right]\right|=$ 0 and $\left|\left[N_{4}, M\right]\right|=4 n_{4}$. Since no major vertex is adjacent with both 4-vertex and 5-vertex, $\left|\left[N_{4}, M_{4}\right]\right|=4 n_{4}$. Each vertex in $M_{4}$ can be adjacent with at most three 4-vertices. Thus $\left|M_{4}\right| \geq$ 
$\frac{4 n_{4}}{3}$. Also $\left|\left[N_{5}, M_{5}\right]\right| \geq 2\left(n_{5}-n_{3}\right)+4 n_{3}-k=2 n_{3}+2 n_{5}-k$. Since each major vertex in $M_{5}$ can be adjacent with at most four 5-vertices, $\left|M_{5}\right| \geq \frac{2 n_{3}+2 n_{5}-k}{4}$. Now $|M| \geq\left|M_{2}\right|+\left|M_{3}\right|+$ $\left|M_{4}\right|+\left|M_{5}\right|+\left|M_{35}\right| \geq 2 n_{2}+n_{3}-\frac{k}{2}+\frac{4}{3} n_{4}+k+\frac{2 n_{3}+2 n_{5}-k}{4}$. Thus $n_{6} \geq 2 n_{2}+\frac{3}{2} n_{3}+\frac{4}{3} n_{4}+\frac{1}{2} n_{5}+\frac{k}{4}$. Now $2 m=2 n_{2}+$ $3 n_{3}+4 n_{4}+5 n_{5}+6 n_{6}=5 n-3 n_{2}-2 n_{3}-n_{4}+n_{6} \geq 5 n-$ $3 n_{2}-2 n_{3}-n_{4}+2 n_{2}+\frac{3}{2} n_{3}+\frac{4}{3} n_{4}+\frac{1}{2} n_{5}+\frac{k}{4}=5 n-n_{2}-\frac{1}{2} n_{3}+$ $\frac{1}{3} n_{4}+\frac{1}{2} n_{5}+\frac{k}{4}$. Thus $m \geq \frac{5}{2} n-\frac{1}{2} n_{2}-\frac{1}{4} n_{3}+\frac{1}{6} n_{4}+\frac{1}{4} n_{5}+\frac{k}{8}$ and hence $m>\frac{5}{2} n+\frac{3}{2}$ if $4 n_{4}+6 n_{5}-12 n_{2}-6 n_{3}+3 k>36$.

\section{Observations}

The Vizing's bound on the size of 6-critical graphs is $m \geq$ $\frac{5}{2} n+\frac{3}{2}$.

- If $G$ is a 6-critical graph in which each 3-vertex is adjacent with a 5-vertex and each 4-vertex is adjacent with another 4-vertex, then the improvement in Vizing's bound on size is attained in the following cases.

$$
\begin{aligned}
& (*) n_{2}=0 \text { and } n_{3} \geq 2 \\
& (*) n_{2}=0 \text { and } n_{4}>6 \\
& (*) n_{2}=0 \text { and } n_{5}>6 \\
& (*) n_{2} \neq 0 \text { and } n_{2}<\frac{5}{2} n_{3}+\frac{n_{4}}{2}+\frac{n_{5}}{2}-3
\end{aligned}
$$

- If $G$ is a 6-critical graph such that each 4-vertex is adjacent with another 4-vertex and no 3-vertex is adjacent with a 5-vertex, then the improvement in Vizing's bound on size is attained in the following cases.

$$
\begin{aligned}
& (*) n_{5}>6+2 n_{2}+n_{3}-n_{4} \\
& (*) n_{2}=n_{3}=0 \text { and } n_{4}+n_{5}>6
\end{aligned}
$$

- If $G$ is a 6-critical graph such that every 3-vertex is adjacent with a 5-vertex, no 4-vertex is adjacent with any minor vertices and no major vertex is adjacent with both 4-vertex and 5-vertex, then the improvement in Vizing's bound on size is attained in the following cases.

$$
\begin{aligned}
& (*) n_{5}=0 \text { and } n_{4}>9+3 n_{2}+\frac{3}{2} n_{3} \\
& (*) n_{5} \neq 0 \text { and } n_{5}>6+2 n_{2}+n_{3}-\frac{2 n_{4}}{3}
\end{aligned}
$$

- An existing bound on the number of major vertices of edge chromatic 6-critical graphs given in R3 is $n_{6} \geq$ $2 n_{2}+\frac{3}{2} n_{3}+\frac{2}{3} n_{4}+\frac{1}{2} n_{5}$.

The following table lists the theorems which improve this bound.

\begin{tabular}{|l|l|l|}
\hline Theorem & $\begin{array}{l}\text { Obtained bound on the number of } \\
\text { major vertices }\left(n_{6}\right) \text { of edge } \\
\text { chromatic 6-critical graphs }\end{array}$ & $\begin{array}{l}\text { The quantity } \\
\text { by which the } \\
\text { improvement } \\
\text { is attained }\end{array}$ \\
\hline Theorem 3.1 & $n_{6} \geq 2 n_{2}+\frac{9}{2} n_{3}+\frac{3}{2} n_{4}+\frac{1}{2} n_{5}$ & $\frac{3}{3} n_{3}+\frac{5}{6} n_{4}$ \\
\hline Theorem 3.2 & $\begin{array}{l}n_{6} \geq 2 n_{2}+\frac{3}{2} n_{3}+\frac{3}{2} n_{4}+\frac{1}{2} n_{5}+\frac{k}{4} \\
\text { where } k=\left|\left[M_{35}, N_{3}\right]\right|\end{array}$ & $\frac{5}{6} n_{4}+\frac{k}{4}$ \\
\hline Theorem 3.3 & $n_{6} \geq 2 n_{2}+\frac{3}{2} n_{3}+\frac{4}{3} n_{4}+\frac{1}{2} n_{5}+\frac{k}{4}$ & $\frac{2}{3} n_{4}+\frac{k}{4}$ \\
\hline
\end{tabular}

\section{Conclusion}

This paper gives new bounds on the number of major vertices and the size of three different classes of edge chromatic 6-critical graphs. Also, this paper lists the cases in which improvement of Vizing's bound is attained. Also improvement on Fiorini's bound on the number of major vertices is obtained in all the three classes of edge chromatic 6-critical graphs.

\section{References}

[1] S. Fiorini , Some remarks on a paper by Vizing on critical graphs, Math. Proc. Comb. Phil. Soc., 77(1975), 475-483.

[2] S. Fiorini and R.J.Wilson, Edge colourings of graphs, Research Notes in Mathematics, 16(1977), Pitman, London.

[3] I.T. Jakobsen, On critical graphs with chromatic index 4, Discrete Math., 9(1974), 265-276.

[4] K. Kayathri, On the size of edge-chromatic critical graphs, Graphs and Combinatorics, 10(1994), 139-144.

[5] Limin Zhang, Every planar graph with maximum degree 7 is of class 1, Graphs and Combinatorics, 16(2000), 467-495.

[6] Limin Zhang, Wenjun Shi, Xianzhen Huang, Guangrong Li, New results on chromatic index critical graphs, Discrete Mathematics, 309(2009), 3733-3737.

[7] Rong Luo, Lianying Miao and Yue Zhao, The Size of Edge Chromatic Critical Graphs with Maximum Degree 6, Journal of Graph Theory, 60(2)(2009), 149-171.

[8] V.G. Vizing, On an estimate of the chromatic class of a pgraph(Russian), Diskret. Analiz, 3(1964), 25-30.

[9] V.G. Vizing, Critical graphs with a given chromatic class(Russian), Diskret. Analiz, 5(1965), 9-17.

[10] V.G. Vizing, Some unsolved problems in Graph Theory, Uspekhi Mat. Nauk, 23(1968), 117-134; Russian Math. Surveys, 23(1968), 125-142.

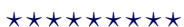

$\operatorname{ISSN}(\mathrm{P}): 2319-3786$

Malaya Journal of Matematik

$\operatorname{ISSN}(\mathrm{O}): 2321-5666$

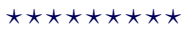

